\title{
Être adulte en sortant de structures d'Aide sociale à l'enfance, le capital social au cœur de la définition de l'autonomie
}

Aude Kerivel

\begin{abstract}
I I LA FIN de la jeunesse contemporaine a souvent été décrite comme n'étant plus délimitée par des « rites de passage », la situation des jeunes placés fait à ce sujet figure d'exception. En effet, la « fin de prise en charge », qui correspond au départ du lieu de placement mais aussi à l'arrêt des allocations financières et de l'accompagnement éducatif à 18 ans (dans certains cas à 21 ans lors des signatures de contrat jeune majeur), constitue indéniablement un rite de passage. Bien loin de la possibilité d'une «expérimentation sans fin ${ }^{1}$ », pour reprendre l'expression de Michel Bozon, ces jeunes se trouvent face à une "rupture nette» qui les plonge dans la vie d'adulte. Une vie d'adulte fortement impactée au vu des enquêtes, notamment de l'INED qui constate que « les personnes ayant connu des événements douloureux dans leur enfance et leur jeunesse ont une probabilité plus élevée de se retrouver sans logement autonome à l'âge adulte ${ }^{2}$ ».
\end{abstract}

Aude Kerivel est chercheure, sociologue, chargée de recherche au Laboratoire d'études, de recherche et de formation en action sociale (LERFAS) de l'ITS de Tours.

1. Michel Bozon. «Des rites de passage aux "premières fois". Une expérimentation sans fins », Agora débats/jeunesses, n ${ }^{\circ} 28,2002$, p. 22-33.

2. Jean-Marie Firdion, «Influence des événements de jeunesse et héritage social au sein de la population des utilisateurs des services d'aide aux sans-domicile », Économie et statistique, $\mathrm{n}^{\circ} 391-392,2006$, p. 85. 
Les professionnels des structures d'Aide sociale à l'enfance semblent donc avoir des raisons de s'inquiéter lorsque les résultats de cette même enquête montrent que les personnes ayant été "placées » sont largement surreprésentées parmi les populations sans domicile (estimées à $23 \%$ dans cette enquête de l'INED, à comparer à $2 \%$ en population générale logée), en particulier parmi les plus jeunes (35\% des 18-24 ans). Une période correspondant au moment de la sortie des dispositifs de l'Aide sociale à l'enfance.

Un constat qui engendre différentes interprétations. Sont-ce les « événements familiaux graves » pesant sur «l'élaboration de structures cognitives et de principe d'organisation » et " altérant l'estime de soi », pour reprendre les propos de Jean-Michel Firdion ${ }^{3}$, ou encore l'expérience de placement, le stigmate et la séparation engendrée par celui-ci, si nous nous situons dans une perspective goffmanienne, qui pèsent le plus dans le fait de faire expérience de la rue ? Telles sont les questions que nous pouvons nous poser.

De plus, si le rapport de l'INED montre qu'environ $40 \%{ }^{4}$ des jeunes sans domicile ont connu le placement dans un foyer, en famille d'accueil ou dans une institution spécialisée, aucune enquête ne nous permet de connaître le pourcentage de jeunes placés qui se retrouvent sans domicile fixe. Cette donnée statistique peut être lue de différentes manières : tous les enfants placés ne deviennent pas sans domicile fixe, et $60 \%$ des jeunes sans domicile fixe n'ont pas été des enfants placés. Qu'est-ce qui fait qu'un enfant placé se retrouve ou ne se retrouve pas à la rue ? C'est la question qui émerge de ce constat.

La sortie de dispositif d'Aide sociale à l'enfance, qui correspond au moment du passage à l'âge adulte, a donc toutes les raisons d'être cruciale. Il s'agit pour les professionnels de préparer les jeunes à la sortie et donc de les " accompagner vers l'autonomie », pour reprendre l'expression inscrite sur l'ensemble des projets des établissements. Un objectif large et flou qui laisse place à de nombreuses interprétations. Ce moment particulier de la fin du placement et de l'entrée dans la vie d'adulte ne fait pas l'objet de nombreuses études. Pourtant, l'expérience de placement n'encourt pas les mêmes réalités pour l'ensemble des enfants et des jeunes, et nous pouvons faire l'hypothèse que certains éléments avant, puis au moment de la sortie impactent ce moment de la sortie, et parfois la suite de la trajectoire.

3. Ibid., p. 85 .

4. Source : enquête auprès des personnes fréquentant les services d'hébergement ou les distributions de repas chauds, Insee, janvier 2001. 
Notre réflexion repose sur plusieurs terrains d'enquêtes menées dans le cadre de travaux de recherche, mais aussi d'évaluation de politiques publiques ainsi que d'évaluation interne de structures sociales (cf. encadré ci-dessous). Des occasions de rencontrer des jeunes un peu avant ou bien après leur sortie du dispositif, ainsi que les professionnels des différentes structures qui les accompagnent dans ce passage à l'âge adulte. Nous nous référons principalement à deux « expérimentations jeunesse », menées entre 2010 et 2013, visant à « éviter les ruptures de parcours de jeunes sortant de l'Aide sociale à l'enfance et de la protection de la jeunesse » par le biais d'une expérience de mise en œuvre de commissions proposant accompagnement et allocation dans un département d'Ile-de-France, et d'une expérience de colocation dans un département de l'Orne. Nous nous appuyons aussi sur une recherche menée entre 2010 et 2014 sur le devenir des enfants placés en villages d'enfants, devenus adultes. Notre réflexion est également nourrie par l'évaluation interne d'une Maison d'enfants à caractère social (MECS), menée durant la même période.

Durant ces rencontres avec des professionnels et des jeunes, plusieurs constats sont apparus de manière récurrente. D'une part, le sentiment pour les professionnels d'une impuissance face à une sortie précoce pour «des jeunes qui ne sont pas encore autonomes » et, d'autre part, l'évocation d'un isolement chez de nombreux jeunes rencontrés nous ont amené à considérer le « capital social ${ }^{5}$ » comme la possibilité d'être " autonome ». Nous défendons ici l'idée selon laquelle l'autonomie - terme à définir - ne doit pas, dans sa définition, se centrer sur « un projet individualisé défini et stable » mais sur l'acquisition d'un capital social dense et solide.

Nous commencerons par montrer que l'injonction à l'autonomie à 18 ans (ou à 21 ans) continue de positionner des jeunes anciennement placés dans une posture hors norme : être adulte avant l'âge. En effet, le rite de passage à l'âge adulte que constitue la fin du placement empêche l'expérimentation comprise dans la définition même de la jeunesse. Puis, en reprenant différents éléments dégagés des enquêtes, nous tenterons de mettre en lumière que les changements de lieu de vie, conséquences du placement, rendent difficiles la création et le maintien de liens.

Au moment de la sortie, si la vulnérabilité économique est indéniable, l'isolement est un véritable problème nommé par les sortants de dispositifs. À l'inverse, un lieu ou un groupe qui perdure facilite la

5. Nous utilisons ici la notion de «capital social» telle que Bourdieu l'emploie dans sa théorie de l'économie des pratiques (Pierre Bourdieu, La distinction. Critique sociale du jugement, Paris, Minuit, 1979). 
solidité des liens. Les constitutions de lien se font à plusieurs occasions et dans plusieurs endroits - les loisirs étant un endroit privilégié.

Le projet individuel n'existant pas sans collectif, nous faisons l'hypothèse que le collectif doit venir en amont du projet individuel (s'essayer au projet collectif est nécessaire pour pouvoir envisager un projet individuel. Et le projet individuel ne peut se faire sans ressource sociale, l'autre étant indéniablement témoin des engagements pris).

\section{UNE RÉFLEXION À PARTIR DE PLUSIEURS TERRAINS}

Deux évaluations d'expérimentations menées entre 2009 et 2014 dans le cadre du Fonds d'expérimentation jeunesse (FEJ), du ministère de la Jeunesse :

- expérimentation d'un partenariat pour éviter les ruptures de parcours: cette première expérimentation, porté par le conseil général d'un département de la région parisienne, visait à « éviter les ruptures de parcours des jeunes sortant des dispositifs d'Aide sociale à l'enfance (ASE) et de Protection judiciaire de la jeunesse (PJJ) ». Près de 200 jeunes entre 16 et 21 ans, repérés par l'ASE, la PJJ, les missions locales et les services de prévention du secteur, ont été concernés par la démarche ;

- expérimentation de colocation : cette deuxième expérimentation, portée par une association d'aide au logement, visait à proposer des logements en colocation à des jeunes sortant de dispositif ASE et PJJ dans une petite commune de milieu plutôt rural. Une quinzaine de jeunes entre 18 et 21 ans ont partagé une des colocations mises en place par l'association.

Ces deux évaluations de politiques publiques ont été l'occasion de rencontrer l'ensemble des professionnels des structures concernées, mais surtout des jeunes juste avant ou juste après ce rite de passage que constitue la fin de prise en charge.

Une recherche: "Que sont devenus les anciens enfants placés en villages d'enfants?"

Notre enquête a été effectuée entre 2011 et 2014 auprès d'anciens enfants accueillis dans quatre villages de la fondation Action Enfance (deux en région parisienne et deux en région Centre), arrivés après 1981 et sortis avant 2010. L'objectif de l'enquête par questionnaire et entretien était d'interroger l'expérience du placement a posteriori, ainsi que la trajectoire de la sortie à aujourd'hui. Notre échantillon, proche de la population mère, est constitué de 122 adultes restés en moyenne 8,7 ans dans un village d'enfants d'Ile-de-France ou de la région Centre entre 1982 et 2007. Ils ont en moyenne 31 ans et sont pour $58 \%$ d'entre eux des femmes.Cette recherche a été l'occasion de rencontrer un certain nombre de professionnels des structures et surtout des adultes étant sortis depuis plus ou moins longtemps. 
$\ldots / \ldots$

\section{Une évaluation interne d'une MECS}

Dans le cadre de l'évaluation interne d'un dispositif innovant d'accueil des « incasables ${ }^{6} »$ par les professionnels de l'Aide sociale à l'enfance. À mi-chemin entre la famille d'accueil et le foyer collectif, cette structure accueille une vingtaine de jeunes qui restent en moyenne un an et demi, c'est peut-être cette courte durée qui amène à faire de la question de l'accompagnement à la sortie un enjeu particulier, «travaillé dès l'entrée du jeune dans le dispositif » aux dires des équipes.Cette évaluation interne a été l'occasion de rencontrer l'ensemble des professionnels des équipes pluridisciplinaires.

\section{L'INJONCTION À L'AUTONOMIE : LA CONTINUITÉ D'UN STIGMATE}

\section{|| Jeunes sortant de dispositif : pas le temps pour l'expérimentation}

Les nombreuses recherches sur « la jeunesse »s'accordent sur le constat d'un allongement de cet âge de la vie ${ }^{7}$ depuis son apparition dans les années 1970 . Les « rites de passages ${ }^{8}$ », tels que la première communion, la conscription pour les hommes et le mariage pour les femmes qui marquaient le passage à l'âge adulte, ont cédé la place «à une transition plus progressive ${ }^{9} »$, un temps $\left\langle\mathrm{d}^{\text {'expérimentation }}{ }^{10} »\right.$. Si l'allongement des études a été à l'origine de l'apparition de cette catégorie, l'évolution des liens parents-enfant aurait été propice à une cohabitation plus longue ${ }^{11}$. Il n'est pas toujours facile de savoir ce qui relève de la contrainte ou du choix pour les jeunes, dans un contexte où l'autonomie financière est grandement conditionnée par l'obtention d'un emploi stable et à temps plein. L'évolution de la jeunesse peut se résumer en quelques statistiques. Aujourd'hui en France, cinq ans séparent

6. Utilisé par les acteurs de la protection de l'enfance, le terme « incasable » est presque devenu une catégorie à part entière. En 2006, l'Observatoire national de l'enfance en danger (ONED) lance un appel d'offres thématique intitulé « Les mineurs dits incasables », Barreyre définissant les jeunes dits « incasables» comme une population à la limite des institutions (Jean-Yves Barreyre et Patricia Fiacre, "Quand le projet fait fuir le sujet», Vie sociale, $\mathrm{n}^{\circ} 4,2010$, p. 2).

7. Olivier Galland, Sociologie de la jeunesse, Paris, Armand Colin, 4 e éd., 2007.

8. David Le Breton, «Les jeunes prennent leur autonomie par le piercing », lemonde.fr, 25 mars 2004.

9. Michel Bozon, op. cit.

10. François Dubet, Sociologie de l'expérience, Paris, Le Seuil, 1994.

11. François de Singly, Libres ensemble. L'individualisme dans la vie commune, Paris, Nathan, 2000. 
la fin de la formation et le premier $\mathrm{CDI}^{12}$. Les jeunes obtiennent donc un emploi stable en moyenne à 28 ans ${ }^{13}$. Le premier emploi n'étant pas nécessairement synonyme d'une indépendance financière, puisqu'un jeune sur deux continue de percevoir une aide de ses parents, même après la cohabitation avec ceux-ci qui se termine autour de 25 ans. Une « vie autonome sans risque ${ }^{14}$ » pour ces jeunes qui continuent, pour $20 \%$ d'entre eux, de rentrer chez leurs parents tous les week-ends pendant au moins six mois, et pour les $14 \%$ qui se réinstallent chez leurs parents dans les cinq ans après les avoir quittés. Dans ce contexte, nous voyons que cette période d'expérimentation, de tâtonnement, de possibilité de prendre le temps de changer d'orientation, d'essayer une formation puis une autre, d'aller à l'étranger pour compléter son cursus, de se tromper, d'échouer est fortement conditionnée par ce soutien familial.

Après une enfance « à part », vécue comme « hors normes ${ }^{15}$ ", les jeunes sortant des dispositifs d'Aide sociale à l'enfance ou de Protection judiciaire de la jeunesse se retrouvent à devoir faire face au rite de passage à l'âge adulte qu'est la fin de placement, qui les positionne dans une situation bien différente de cet "état » défini comme " durable » qu'est la jeunesse et de la transition plus progressive au statut d'adulte ${ }^{16}$. Pas le temps pour le tâtonnement ou l'expérimentation, puisque la fin de la prise en charge entraîne un basculement prématuré dans la vie d'adulte à 18 ans. Lorsque sur certains territoires ${ }^{17}$ l'accompagnement se poursuit jusqu'à 21 ans dans le cadre de contrats jeunes majeurs, c'est souvent à la condition d'un projet professionnel bien défini et entamé, et non pour permettre d'allonger un peu ce temps de jeunesse pour ceux qui ne se sentent pas prêts à être adultes.

L'évaluation du dispositif de partenariat visant à éviter les ruptures de parcours a permis de rendre visible « le besoin de temps d'expérimentation », un temps pour rebondir après un échec scolaire, se réorienter, entrer dans la vie active ou s'approprier un projet, un temps

12. Livre vert Synthèse des propositions de la Commission de concertation sur la politique de la jeunesse, 2009, p. 11.

13. Économie et statistique, $\mathrm{n}^{\circ} 337-338,2000$.

14. Michel Bozon, op. cit.

15. Les différents entretiens menés avec les jeunes des dispositifs d'aide sociale à l'enfance $\mathrm{du}$ Val-de-Marne et les jeunes adultes anciennement accueillis en villages d'enfants font état du stigmate lié au fait de ne pas avoir vécu « comme tout le monde » avec ses parents.

16. Michel Bozon, op. cit.

17. Comme l'observe Flore Capelier dans le rapport de l'ONED « L'accompagnement vers l'autonomie des jeunes majeurs » (2015), l'accompagnement des jeunes entre 18 et 21 ans pris en charge par les départements présente de fortes hétérogénéités : "L'utilisation du verbe pouvoir et non devoir par le législateur [...] conduit la plupart des départements à considérer que les prestations proposées par le service de l'Aide sociale à l'enfance en direction de ce public sont facultatives » (p. 24). 
pour choisir et s'engager dans une formation, ou parfois pour régler un souci qui fait blocage. Ces jeunes manquent de temps pour expérimenter comme n'importe quel jeune, mais manquent également de moyens pour devenir adultes et « se projeter dans une temporalité ${ }^{18}$ », pour reprendre la définition de François Dubet, dans une situation particulièrement vulnérable et incertaine.

\section{|| L'impuissance des professionnels}

Alors que se développe une rhétorique « de la mobilité », « de la capacité d'adaptation » et « de l'innovation » qu'interroge notamment Hartmut Rosa ${ }^{19}$, associée à la valorisation d'un " idéal de jeunesse », il est demandé aux jeunes sortant des dispositifs d'Aide sociale à l'enfance d'être stables, de faire des choix et de ne pas perdre de temps. Loisirs, expériences hors travail traditionnel sont perçus comme non indispensables et non prioritaires ${ }^{20}$. Une injonction contradictoire est faite aux futurs sortants des services de l'Aide sociale à l'enfance : tandis que l'Éducation nationale valorise le fait d'aller le plus loin possible dans les études, les professionnels de l'éducation spécialisée, contraints par le temps, valorisent les études courtes et l'insertion professionnelle rapide.

« Je suis parti à 15 ans à Loches, puis à 17 ans à Grenoble, puis je dépendais de l'antenne extérieure, jusqu'à 18-20 ans, moins de 21 ans, parce que je me rappelle à l'époque j'avais raté un concours, en fait j'avais plusieurs diplômes à passer, j'en ai loupé un et ils l'ont très mal pris, parce qu'ils estimaient que ça coûtait cher juste pour le matos de ski, l'école, et donc ils ont continué à me payer l'école, mais ils ont arrêté de me payer ma subvention, donc du coup j'ai dû trouver un boulot et je me rappelle que je travaillais la nuit et j'allais en cours la journée. C'était une période assez difficile, encore à l'époque j'étais jeune, je récupérais bien, ça se faisait » (Damien, 30 ans, anciennement placé en village d'enfants).

Lorsque des études après 18 ans sont envisagées, les jeunes n'ont pas le droit à l'erreur, pas la possibilité d'expérimenter. Les professionnels se trouvent dans une double impasse. L'échéance de la fin de leur

18. David Le Breton dans P. Caillé, La quête de reconnaissance : nouveau phénomène social total, Paris, La Découverte, 2007, p. 48.

19. Hartmut Rosa, Accélération. Une critique sociale du temps, Paris, La Découverte, 2010.

20. Lors de la commission du projet de «partenariat pour éviter les ruptures de parcours des jeunes sortant de l'Aide sociale à l'enfance ", les professionnels (de l'ASE, de la PJJ, des missions locales, des associations de prévention) ont débattu, afin de déterminer si les projets proposés par les jeunes étaient « finançables » ou non. Si les projets professionnels (à la condition que ceux-ci soient stables et que le jeune n'ai pas trop changé ses projets) étaient unanimement considérés comme " pertinents » et donc financés, il n'en était pas de même pour les projets de continuité de loisirs, de séjour linguistique ou de brevet non professionnel tel que le BAFA. 
mission à la majorité du jeune et leur défaut de pouvoir d'action quant à traiter la question de l'insertion professionnelle les renvoient à leur impuissance.

\section{| L'injonction à l'autonomie}

Dans ce contexte peu confortable, les professionnels se focalisent sur un objectif : celui de l'accès à l'autonomie, qui se décline en « projet individuel », « parcours individuel », « projet d'accompagnement personnalisé ». Lors de nos différentes enquêtes, un discours revenait sans cesse dans la bouche des professionnels : «Les jeunes ne sont pas prêts, ne sont pas autonomes. ")

«Les jeunes qui sont en foyer, en famille d'accueil, ils ne sont pas autonomes. Quand ils sortent, à 18 ans, ils ne sont pas préparés » (responsable de service structure ASE) ${ }^{21}$.

«Quand on a vécu en foyer, on est déconnecté : les jeunes n’ont jamais fait leurs courses tout seuls, on les accompagne à leurs rendez-vous médicaux, ils mangent en collectivité, ils sont peut-être encore moins autonomes que des jeunes qui quittent leur famille, et pourtant ils doivent partir plus tôt » (responsable de service ASE).

Ce constat est rapidement remis en cause dès qu'un travail plus approfondi est initié. En effet, ces jeunes, ayant déjà pour la plupart une expérience professionnelle, sachant gérer un certain nombre de domaines de leur vie dont ne s'occupent pas leur famille, sont perçus au final comme plus autonomes.

Cette contradiction, conséquence du flou de la définition et pourtant à la source d'une injonction forte faite aux jeunes, demande réflexion. Le nouveau dictionnaire critique d'action sociale, publié en 2006 sous la direction de Jean-Yves Barreyre et Brigitte Bouquet, a rassemblé « 300 mots-clefs pour comprendre vraiment l'action sociale dans ses méthodes ». Parmi les définitions proposées, celle d'autonomie, désignant « la capacité, la liberté, le droit d'établir ses propres lois et la capacité de se gouverner soi-même ». Utilisée pour désigner l'autonomie de la cité chez les philosophes grecs, la notion est reprise pour parler de « l'individu libre », en opposition à celui qui se soumet. « En dehors du champ politique, la notion d'autonomie [est] souvent considérée comme n'ayant pas de réel contenu conceptuel »; l'autonomie ne signifie pas « suppression de dépendance » « puisqu' à l'intérieur du système, on ne peut être autonome que par ou à travers des dépendances ». Edgar Morin considère quant à lui l'autonomie de l'individu

21. Extraits d'entretiens individuels menés avec les cadres des différentes organisations, membres de la commission ROAD. 
dans le paradoxe de sa dépendance à l'environnement. Dès lors, l'autonomie serait «l'autogestion équilibrée des multiples réseaux et chaînes de dépendances que l'on entretient entre individus, groupes, institutions, objets, réels ou irréels ${ }^{22} \gg$. La définition se termine ainsi : « L'autonomie resterait toujours relative, dépendante de la contingence réciproque du même et de l'autre, positionnée dans une trame relationnelle avec autrui et l'environnement. Seul importerait le jeu contraire et complémentaire de l'autonomie et de l'hétéronomie, de la dépendance et de l'autonomie, couples liés et féconds ${ }^{23}$. » Et si c'était de ressources dont les jeunes avaient besoin, plus que d'autonomie ? C'est la question que nous pouvons nous poser.

L'évaluation du projet de partenariat pour éviter les ruptures de parcours des jeunes sortant des dispositifs de l'Aide sociale à l'enfance a mis en avant le fait que ces jeunes avaient majoritairement déjà une ou plusieurs expériences d'emploi entre 18 et 21 ans (ce qui les rend au final peut être plus précoces que les jeunes de leur âge), mais leur vulnérabilité apparait lorsqu'ils sont « entre deux emplois » :

«En fait, j'étais en difficulté. C'est des difficultés financières. Une aide sur l'assurance auto et une aide sur une ALJT [Association logement jeunes travailleurs] et j'ai eu le droit à une prise en charge par mes éducateurs en milieu ouvert... J'étais en formation, j'étais payé très peu. Une demande pour l'assurance du permis m'a beaucoup aidé pour me déplacer pour le travail. En foyer de jeunes travailleurs. Pour un micro-ondes et un frigo... Je sais pas, c'était bien ça que je demandais » (Bakari, 19 ans, en fin de prise en charge ASE du Val-de-Marne).

Face à cette injonction forte à l'autonomie et confrontés à la fin de prise en charge précoce, les jeunes se retrouvent maintenus dans cette situation « hors normes » qui était déjà la leur auparavant. Certains voient toutefois la fin du placement comme la possibilité d'être perçus autrement que comme « enfants de la DASS ${ }^{24}$ ».

Skander, 18 ans, bénéficiant de l'expérimentation visant à éviter les ruptures de parcours des jeunes sortant de l'ASE, vient d'être orienté dans une colocation à la suite de sa fin de placement et dans le cadre d'un contrat jeune majeur. Il vient de quitter sa famille d'accueil, dans laquelle il a vécu pendant seize ans. Il échange avec un autre jeune dans la même situation :

22. Ibid., p. 61.

23. Ces éléments sont issus de la définition donnée dans Jean-Yves Barreyre, Brigitte Bouquet (sous la direction de), Nouveau dictionnaire critique d'action sociale, Paris, Bayard, 2006.

24. Le terme « enfant de la DASS », bien que désuet depuis 2007, a été utilisé par les anciens placés en villages d'enfants, même après cette période, lors des entretiens. 
Skander : «Le fils de ma famille d'accueil, il a 34 ans et il vit toujours chez ses parents.

John : Faut du courage.

Skander : Non ce n'est pas du courage, c'est le gars qui n'a pas d'activité extérieure, c'est le gars à papa et à maman. Il n'a pas le permis, il a rien. John : Si ça lui convient.

Skander : Oui ça lui convient, il n'est pas contre. Si tu veux, c'est bien dans un sens car il taffe, du coup il met de l'argent de côté, il n'a pas trop à dépenser. »

Si, de fait, les jeunes pris en charge par l'Aide sociale à l'enfance, parce qu'ils basculent prématurément dans le monde des adultes, ont besoin d'acquérir de «l'autonomie », nous verrons qu'il convient de revenir sur les choix qui peuvent être faits pour définir ce concept. C'est à partir des besoins et potentiels des jeunes repérés sur nos quatre terrains que nous axerons notre définition.

\section{EXPÉRIENCE DE PLACEMENT ET ISOLEMENT}

\section{|| La vulnérabilité comme héritage}

Le point commun de l'ensemble des jeunes sortant des services d'Aide sociale à l'enfance et de protection de la jeunesse, c'est qu'ils se trouvent dans une «zone de vulnérabilité ${ }^{25}$ ». Les enquêtes sur lesquelles nous nous appuyons pour cet article confortent les travaux de Donzelot ${ }^{26}$ : les parents sont majoritairement exclus du monde de l'emploi et/ou appartiennent aux classes sociales populaires.

Jean-Marie Firdion fait également ce constat et explique que ces « événement familiaux graves » vécus par les enfants placés « ne sont pas indépendants du contexte socio-économique ${ }^{27} \gg$. Notons par ailleurs un nombre non négligeable de parents décédés ${ }^{28}$ avant, mais aussi pendant le placement.

25. Selon Robert Castel (Les métamorphoses de la question sociale, une chronique du salariat, Paris, Fayard, 1995), ce terme correspond à une fragilisation des statuts dans la sphère de l'emploi et dans la sphère sociale.

26. Jacques Donzelot, La police des familles, Paris, Minuit, 1977.

27. Jean-Marie Firdion, op. cit., p. 85.

28. Sur la cohorte de jeunes sortant de l'Aide sociale à l'enfance et de la Protection judiciaire de la jeunesse, $10,9 \%$ des mères et $14,4 \%$ des pères sont décédés avant ou pendant le placement. Concernant les anciens jeunes placés en villages d'enfants, à la sortie $16 \%$ des jeunes avaient perdu leur mère et $21 \%$ leur père. Ces différents éléments font état d'une santé précaire des parents dans des contextes vulnérables. 
La vulnérabilité économique des jeunes au moment de leur sortie est liée à l'âge et au fait qu'ils ne sont pas encore véritablement insérés sur le marché du travail, mais surtout ils ont hérité de la vulnérabilité économique de leurs parents. Celle-ci s'accompagne bien souvent d'une vulnérabilité sociale causée par la précarité ou l'absence d'emploi, mais aussi du stigmate associé à une situation ou pratique déviantes (à l'origine du placement) puis au fait de voir son/ses enfants placés, parfois de liens déjà distendus avec la famille d'origine ${ }^{29}$.

L'expérience même de placement des enfants disqualifie les familles, pour reprendre Serge Paugam ${ }^{30}$ qui utilise le terme de « disqualification sociale » pour décrire les phases qui font basculer de la précarité à l'exclusion. Selon lui, « la disqualification sociale est donc avant tout une épreuve, non pas seulement en raison de la faiblesse des revenus ou de l'absence de certains biens matériels, mais surtout en raison de la dégradation morale que représente dans l'existence humaine l'obligation de recourir à l'appui de ses semblables et des services d'action sociale pour obtenir de quoi vivre dans des conditions décentes ». Dans ce contexte, les jeunes sortant de l'Aide sociale à l'enfance ont toutes les chances de n'hériter de leurs parents ni d'un capital économique (patrimoine, aide financière) ni d'un capital social ( « ressources liées à la possession d'un réseau durable de relations d'interconnaissance et d'inter-reconnaissance ${ }^{31} \gg$ ) leur permettant de trouver un logement, un stage ou un emploi au moment de leur sortie du dispositif.

\section{|| Expérience de placements multiples et isolement}

Mais pour comprendre les fins de placement, il s'agit de revenir sur ce qui s'est joué pendant le placement lui-même, ou plutôt pendant les placements. Lorsqu'un des 48820 enfants ou jeunes ${ }^{32}$ quitte un établissement de l'Aide sociale à l'enfance, il y est resté en moyenne 13 mois. Notre échantillon est représentatif des disparités entre établissements. En effet, si les enfants demeurent en moyenne 18 mois dans les MECS, 13 mois dans les lieux de vie et 6 mois dans les foyers de l'enfance, ils

29. Les contacts distendus avec la famille élargie s'expliquent parfois par une expérience de placement des parents eux-mêmes lorsqu'ils étaient enfants. Si ce cas n'est pas majoritaire, il existe et permet d'en rendre compte.

30. Serge Paugam, La disqualification sociale, Paris, Puf, 1991.

31. Pierre Bourdieu, « Le capital social », Actes de la recherche en sciences sociales, 1980, p. 2-3.

32. Thierry Mainaud, « Les établissements et services en faveur des enfants et adolescents en difficulté sociale. Activité, personnel et clientèle au 15 décembre 2008 », Direction de la recherche, des études, de l'évaluation et des statistiques, document de travail, série Statistiques $n^{\circ} 173$, septembre 2012. 
restent en moyenne 5 ans dans les villages d'enfants. En considérant que la durée moyenne de la prise en charge ASE est de 7 ans, nous pouvons dire que les jeunes, au moment de leur sortie, ont été placés dans 5 à 6 établissements. Une estimation minimum si nous la confrontons à l'expérience des jeunes eux-mêmes :

« Donc après je suis resté à Cesson jusqu'à l'âge de 13 ans, après j’ai été au foyer d'Évry, qui appartenait toujours au MVE [Mouvement villages d'enfants], après j'ai fait le foyer de Paris, un petit temps, après j'ai fait le..., qui était un foyer de filles en fait, mixte, en fait c'était mixte : des filles de plusieurs villages. Ils avaient construit ce petit foyer à Paris, en fait Paris même, Paris 19e, après euh, comme j'étais un sale môme, on m'a mis au foyer à Melun, qui là par contre, qui était un foyer de filles à $100 \%$, il y avait qu'un garçon, qui était avec moi au village de Cesson, qui n'était là que le week-end, donc je suis venu là en tant que deuxième garçon, mais à temps plein entre guillemets. Donc c'est devenu après un foyer mixte garçons-filles. Après le foyer à Melun, bah j'ai pris un appartement pendant un an » (Simon, 41 ans, ancien placé en village d'enfants).

Même dans ces cas de jeunes accueillis en village d'enfants sur des temps bien plus longs que la moyenne ( 8 ans en moyenne pour notre échantillon), il est intéressant d'entendre cette expérience. Fréquemment, les jeunes décrivant leur parcours expliquent qu'ils sont restés quelque temps dans un premier lieu d'accueil et d'orientation, faisant partie de la fondation, même s'il n'est pas nommé comme tel par les jeunes. Puis avant la fin de placement, ils expliquent avoir été placés dans un foyer d'adolescents « afin de préparer leur sortie », pour reprendre les termes des professionnels. Au final, ce sont trois lieux différents même si l'Aide sociale à l'enfance n'en compte un seul. Ces trois lieux viennent s'ajouter bien souvent à une expérience de famille d'accueil en amont de leur placement en village d'enfants.

Les fondations, les associations sont souvent implantées sur plusieurs sites, dans plusieurs villes, parfois même départements. Les changements sont fonction de l'âge de l'enfant, de son projet ou parfois d'une organisation. Si les structures font partie de la même association, ce sont pour l'enfant ou le jeune des lieux physiques différents, où il se trouve entouré d'enfants, de jeunes, d'adultes différents eux aussi. Plus les changements sont fréquents dans la trajectoire de placement et moins les enfants et jeunes ont la possibilité de lier des contacts et des amitiés.

\section{|| L'isolement des professionnels}

Vulnérabilité des familles, placements successifs des jeunes, l'enjeu est important pour les professionnels qui ont pour mission d'accompagner, d'éduquer, de suivre les enfants placés. 
À l'isolement des familles et des jeunes ayant vécu dans plusieurs lieux de placement successifs vient s'ajouter un isolement des professionnels (éducateurs, assistants de service social), qui ont le sentiment de se retrouver seuls pour accompagner les jeunes dont ils sont les référents. Ce constat a été fait notamment lors de l'évaluation des expérimentations visant à éviter les ruptures de parcours des jeunes sortant de l'Aide sociale à l'enfance et de la Protection judiciaire de la jeunesse. En effet, nous avons alors observé une difficulté à se faire aider, à passer le relais, à travailler avec d'autres, ce qui serait perçu comme la faiblesse de ne pas y arriver seul. Dans un contexte où les organisations sont remaniées et sans cesse soumises au risque d'une perte de financement, les professionnels se recentrent sur l'accompagnement, au risque de renforcer la « relation de dépendance assistantassisté $^{33}$ » et d'influencer ce que Serge Paugam nomme une « carrière d'assisté », où chacun tente de s'identifier et de correspondre aux attentes de l'autre.

La difficulté liée au turnover des professionnels vient parfois se cumuler à cet isolement au sein des équipes. Dans certaines structures, les jeunes ont quelquefois plus d'ancienneté que la majorité des professionnels. Quant aux professionnels, ils se retrouvent dans la situation de devoir faire leurs preuves vis-à-vis de leurs collègues en présence des jeunes.

Mais il faut compter aussi avec l'isolement de la structure en ellemême. La difficulté à travailler en partenariat avec les autres structures du territoire est souvent le fait d'une histoire ou chaque structure s'est construite avec le "nous » de l'institution en opposition aux autres institutions. Pourtant, le travail d'équipe est une possibilité d'élargissement du réseau, comme en témoigne un éducateur ayant participé au dispositif expérimental visant à éviter les ruptures de parcours des jeunes par le biais d'un partenariat entre les structures ASE, PJJ, mission locale et prévention spécialisée du secteur.

«Le projet : c'est vraiment un réseau, une jeune cherchait un stage, on nous a dit autour de la table : donnez-nous les candidatures, on va les transmettre... À un moment donné, aujourd'hui, c'est important d'avoir un réseau, et les jeunes qu'on accompagne ils n'ont pas de réseau, leurs parents n'ont pas de réseau. Donc ils n'ont pas de réseau pour job d'été, pour tout en fait. Nous, nos jeunes ils n'ont pas tout ça... ça a été l'occasion d'avoir ce réseau » (éducateur spécialisé ASE du Val-de-Marne).

Les difficultés à travailler en équipe au sein de la structure et en partenariat avec les autres structures entraînent un isolement des

33. Serge Paugam, op. cit. 
professionnels qui impacte nécessairement les jeunes eux-mêmes. Et il apparaît que le regard sur les jeunes change dès lors que les professionnels se sentent appartenir à une équipe elle-même bien insérée dans un réseau de professionnels.

\section{1| Se retrouver seul, être seul : une difficulté formulée}

Au bout de la chaîne, il y a les jeunes qui, au moment de leur sortie du dispositif, évoquent leurs peurs ou leurs incertitudes :

« Je sais pas ce qui va se passer à mes 21 ans » (Joshua, 20 ans, en fin de prise en charge ASE, Val-de-Marne).

« Il me reste quatre mois, je me disais bien qu'un jour ou l'autre ça allait se terminer » (Jasmine, 20 ans, en fin de prise en charge ASE, Val-de-Marne).

Même si parfois cette crainte est mêlée à un sentiment de liberté et d'émancipation, la difficulté de se retrouver seul est très souvent énoncée :

« Au début, j'étais très contente, ça faisait très longtemps que j'avais fait la demande et toutes mes copines étaient parties avant moi. Du coup, je me retrouvais la plus grande du village. Au début, j'étais contente, les cartons et tout ça, je me disais je vais habiter sur Paris, je vais avoir plus de liberté et tout ça, et en fait en arrivant là-bas, ils m'ont posée. Franchement, ça a été trop dur en fait. Je me retrouvais toute seule, à ne connaître personne. Tellement on a été protégés, du coup, on arrive dans un truc, on ne connait pas. On se dit que franchement, on ne sait pas si on va y arriver ou pas. Franchement, j'ai bien été accueillie, mais ça faisait peur. J'ai eu peur de comment ça allait se passer. Du coup, je voulais repartir au village » (Maëlis, 24 ans, ancienne placée en village d'enfants).

« Moi je suis en famille d'accueil depuis l'âge de 3 ans et j'avais besoin d'un peu d'autonomie et j'ai demandé à avoir un appartement, colocation ou pas, ça ne me dérangeait pas. Je pense que c'est même mieux qu'un appartement tout seul. Au niveau du coût premièrement, même si ce n'est pas moi qui paie, je pense que c'est plus intéressant. Au niveau de la relation aux autres. Moi je n'aime pas trop la solitude » (Skander, 19 ans, fin de prise en charge en famille d'accueil, Orne).

La solitude est d'autant plus brutale que ces jeunes ont passé une grande partie de leur vie dans des institutions collectives, constamment entourés :

« C'est juste que je ne suis pas habituée à vivre toute seule. Tout seul, on déprime. Alors quand il y a du monde, on est au moins obligés de se préparer. Sinon je suis perdue. Quand je vivais seule, des fois je ne savais plus quel jour on était, c'était grand, c'était vide, ça résonnait et donc ça me faisait peur, du coup, je sortais tout le temps » (Sana, 19 ans, fin de prise en charge en MECS, Orne). 
Or, si l'institution de l'Aide sociale à l'enfance est du fait de sa position impuissante quant à la possibilité d'accès à l'emploi des jeunes qu'elle accompagne, elle peut avoir un rôle, nous le verrons, dans la construction d'un réseau sur lequel les jeunes pourront s'appuyer au moment de leur sortie et par la suite.

\section{IIE CAPITAL SOCIAL : UN ENJEU}

\section{Autonomie et capital social}

Et si le capital social de l'individu était au centre de l'autonomie, au lieu de la capacité à faire seul un certain nombre d'actions ? C'est l'hypothèse que nous faisons lorsque nous mettons en perspective nos différentes études, et les écarts dans la manière dont les jeunes sortant des dispositifs ASE vivent leur sortie. Pierre Bourdieu définit le capital social comme : «L'ensemble des ressources actuelles ou potentielles d'un agent qui sont liées à un réseau durable de relations plus ou moins institutionnalisées d'interconnaissance et d'inter-reconnaissance ou, en d'autres termes, à l'appartenance à un groupe comme ensemble d'agents qui ne sont pas seulement dotés de propriétés communes mais aussi unis par des liaisons permanentes et utiles ${ }^{34}$. » Pour ces jeunes, qui au moment de leur sortie se trouvent dépourvus de capital économique et dans une situation d'emploi qui a toutes les chances d'être précaire, le capital social est ce qui leur permet de ne pas se retrouver dans une situation de désaffiliation ${ }^{35}$.

Les différentes trajectoires des jeunes que nous avons pu rencontrer au cours de nos enquêtes nous permettent d'affirmer que l'acquisition et le maintien d'un capital social durant la trajectoire de placement impactent positivement la fin du placement et ce moment particulier d'insertion et de passage à l'âge adulte. Trouver un emploi, un logement, être hébergé en cas de problème, trouver un bon médecin, agir face à une situation imprévue, régler un souci qui fait obstacle en prenant de la distance face à celui-ci, avoir des personnes avec qui échanger, qui peuvent conseiller ou à qui l'on peut donner des conseils sont autant d'exemples concrets permis par le capital social. Nous tenterons de montrer, pendant le placement puis au moment de la sortie,

\footnotetext{
34. Pierre Bourdieu, op. cit.

35. Robert Castel, « De l'indigence à l'exclusion, la désaffiliation », dans Jacques Donzelot (sous la direction de), Face à l'exclusion, le modèle français. Paris, Éditions Esprit, 1991, p. 137-168. Robert Castel préfère le terme de désaffiliation à celui d'exclusion. Pour lui, la désaffiliation renvoie au déficit de filiation : « déficit d'inscription dans des liens sociaux et donc de protection rapprochée (par des proches) » et « déficit d'inscription dans des formes collectives de protection et en particulier de la protection issue des collectifs de travail ».
} 
les expériences des jeunes que nous avons pu repérer comme ayant permis la construction d'un capital social mobilisable lors de ce basculement dans le monde des adultes.

\section{| Les ressources le plus souvent évoquées}

Les éléments recueillis auprès des jeunes nous permettent de déterminer des personnes ressources. Nous parlons de ressources mais nous pourrions aussi utiliser le terme de « supports » qui, comme le notait Robert Castel, sont nécessaires aux individus « qui ne tiennent pas debout tout seuls ${ }^{36} \gg$.

Ils nomment en premier les professionnels des institutions qui les ont accompagnés, parfois l'organisation elle-même ou les partenaires de celle-ci. L'éducateur ou l'éducatrice référent qui les a suivis longtemps, avec qui quelquefois ils ont le sentiment d'une relation privilégiée, peut être repéré comme une personne ressource :

« Il y a eu une éducatrice qui venait chez moi, non pas en tant qu'éducatrice, mais elle venait me voir et tout » (Bénédicte, 35 ans, ancienne placée en village d'enfants).

Les parents sont nommés aussi fréquemment. Si plusieurs jeunes évoquent un retour chez les parents à la fin de la prise en charge, celuici est généralement source de déception. Les jeunes repartent bien souvent rapidement, ne se retrouvant pas dans un contexte familial qu'ils avaient eu tendance à idéaliser. Les membres de la famille élargie ne sont que très rarement nommés :

« Je suis parti chez mon père, voir comment ça se passerait avec mon père, ça ne s'est pas bien passé, parce qu'à 18 ans, pourtant je suis vraiment pas, je suis... on est... c'est fusionnel avec mon père, on est, on est vraiment proches, et donc c'est pour ça que j'y suis allé d'ailleurs, et euh il a essayé, j'avais 18 ans, il a essayé de prendre son rôle de père, forcément, bah voilà c'est... ça l'a pas fait longtemps, et euh, j'ai préféré repartir du coup avant que... avant qu'on dégrade notre relation. Ça a pété qu'une fois, j'ai vu qu'une fois, au point où ça allait j'ai dit, j'ai dit voilà, ça a beau être mon père, si une fois il devait lever la main sur moi, je risquerais d'aller trop loin, j'ai fait : non, vaut mieux que je parte maintenant » (Corentin, 24 ans, ancien placé en village d'enfants).

Les frères et sœurs, lorsqu'un lien a perduré pendant la période de placement, notamment dans les cas où les fratries ont été placées ensemble, (en village d'enfants par exemple), sont fortement mobilisés

36. Robert Castel, «Individus par excès, individus par défaut », dans François de Singly, Christian Le Bart, Philippe Corcuff, L'individu aujourd'hui, Presses universitaires de Rennes, p. 294. 
au moment de la sortie. S'ils ne nomment pas les frères et sœurs, les jeunes sortant de villages d'enfants évoquent tous de multiples situations où ils ont soit soutenu, soit été soutenus par les frères et sœurs. L'aînée joue en particulier un rôle important vis-à-vis de ses cadets ${ }^{37}$ :

«Ça s'est bien passé pendant plusieurs années, et après cata... je suis tombé sur une folle, donc du coup séparation, je pensais rester sur M. au départ parce que c'est une région que j'apprécie énormément, puis comme elle n'a pas accepté la séparation, moments difficiles, tendus, du coup je suis parti, je suis parti 15 jours à B., je suis rentré j'avais plus rien. Mais quand je dis plus rien, y avait de la poussière au sol et tout mais y avait plus rien. Donc je suis parti, parce qu'après on a des idées qui traversent la tête, je voulais éviter de faire des boulettes donc et du coup je me suis rapproché de ma sœur qui venait d'avoir un enfant qui avait trois semaines à l'époque, et voilà du coup j'ai soutenu ma sœur etc. Pour me reconstruire finalement » (Damien, 34 ans, ancien placé en village d'enfants).

« Donc euh, mais bon dans les débuts, c'est vrai qu'on se voyait régulièrement, qu'on a... donc celle de P. c'est pareil, pendant toute une époque je prenais sa fille pendant toutes les vacances scolaires, au moins elle restait en contact avec ses cousins et ses cousines, elle adorait venir passer les vacances parce que A. bon c'est... ça change de P. hein, on respire, mais bon voilà, et elle adorait ça quoi. Et tous les étés, toutes les vacances scolaires, ah ouais, mais j'étais famille je suis très famille » (Valérie, 40 ans, ancienne placée en village d'enfants).

Le rapport aux pairs de placement est ambivalent. D'abord, les liens créés avec ces pairs sont souvent conditionnés par la durée du placement dans une même structure et les expériences collectives communes. Ensuite, au moment de la sortie, la volonté de prendre le large entraîne une coupure dans les contacts repris par la suite :

«Quand nous étions petits, nous allions en car voir les animaux de la ferme du village d'Amboise. Donc, on se connaissait avec les enfants d'Amboise. Moi, j'étais du village de Pocé, mais j'avais sympathisé avec des enfants du village d'Amboise, donc j'y allais souvent. Mais ça, c'est à partir du collège car là on était avec d'autres enfants et parmi eux il y avait ceux du village d'Amboise » (Joseph, 27 ans, ancien placé en village d'enfants).

« Ça m'arrive de voir certaines têtes mais c'est rare, c'est tout les troisquatre ans, c'est bref, ça dure une heure, deux heures, on prend un café et chacun reprend sa vie. J'ai pas beaucoup d'échanges avec mes amis de l'époque. C'est très bizarre, enfin j'en discute avec ma sœur, disons qu'on a tous une phase, mais on a tous une phase de pas bien où l'on se retrouve un petit peu, un peu aware, on fait un peu n'importe quoi... On se retrouve dans une phase sans boulot, on galère et y en a qui ont du mal à s'en sortir de cette phase-là » (Damien, 34 ans, ancien placé en village d'enfants).

37. Aude Kerivel, «Être le plus grand d'une fratrie placée : vécu du placement et passage à l'âge adulte des aînés filles et garçons ", à paraître en 2015 dans Politiques sociales et familiales. 
Les amis rencontrés hors de l'institution constituent des amitiés choisies auxquelles font référence plusieurs jeunes. Rencontrés dans le cadre des loisirs ou à l'école, ces amis et groupes d'amis semblent permettre aux jeunes de revendiquer une autre identité que celle « d'enfants placés »:

« J'ai retrouvé des amis à moi à Paris, quand j'étais en formation à Loches. Mon meilleur ami que j'ai retrouvé sur Paris, du coup on est régulièrement ensemble » (Damien, 34 ans, ancien placé en village d'enfants).

« J'ai un copain, ses parents avaient des chevaux, ils habitaient pas très loin du village d'enfants, c'est vrai que c'est un ami qui n'avait rien à voir avec le village d'enfants, je l'ai rencontré à l'école. Ça me faisait du bien d'aller là-haut. J'allais passer quelques après-midi là-haut. Ça me changeait du contexte du village d'enfants. De temps en temps, ça faisait du bien d'aller voir ailleurs. C'est vrai que c'était le seul que je voyais en dehors du village » (Joseph, 27 ans, ancien placé en village d'enfants).

$\mathrm{Au}$ moment de la sortie, d'autres réseaux se constituent. Les rencontres amoureuses et les familles des conjoints (beaucoup plus citées par les jeunes femmes que par les jeunes hommes) semblent être une nouvelle « famille » sur laquelle s'appuient plusieurs jeunes :

« Mes beaux-parents nous ont pas mal aidés, sinon cela aurait été beaucoup plus compliqué, beaucoup plus compliqué. Ils habitent en Seine-et-Marne. Il y a une demi-heure de route et ils font le déplacement sans problème. Si j'ai un souci, ils viennent nous chercher, on sait que l'on peut compter sur eux. Quand on a eu besoin, ils ont été là, heureusement » (Aline, 20 ans, ancienne placée en village d'enfants).

Les premiers emplois, plus rarement nommés, sont aussi des occasions de nouer des amitiés avec des collègues ou supérieurs qui peuvent être ressources pour la suite du parcours professionnel :

« À Grenoble, j'avais un entraîneur de l'équipe du Luxembourg, il était toujours de bon conseil avec moi, ça a été un des piliers avec moi... Dans ma vie je suis toujours en relation avec lui d'ailleurs et des fois ça allait plus loin que le sport, ça m'arrivait d'aller manger dans sa famille, il venait chez moi » (Damien, 34 ans, ancien placé en village d'enfants).

Si nous avons cherché, dans les entretiens que nous avons effectués, à observer le capital social acquis pendant le placement puis au moment de la sortie, c'est également parce que nous avons repéré un besoin et une demande par ailleurs. Comme lors de l'expérimentation visant à éviter les ruptures de parcours, où les professionnels de l'ASE et de la PJJ ont été étonnés que la proposition de parrainage emporte un tel succès.

«C'est moi qui l'ai choisie ma marraine, on s'est vus une fois avec mon éducatrice, et puis ensuite seuls. On se téléphone et on communique par 
mail. Il y avait plusieurs choix, soit un truc financier, j'ai déjà un contrat jeune majeur donc j'ai pas besoin de trucs financiers, donc j'ai choisi une marraine, je voulais quelqu'un avec qui je pouvais parler. C'est un bon projet. Ils ne m'ont pas proposé quelqu'un pour me juger, ou me dire tu fais ça ou ça... mais quelqu'un juste avec qui je vais parler... On s'entend bien, y a pas de souci » (Nadir, 21 ans, sortant de foyer ASE, Val-de-Marne).

\section{Expériences, groupes d'appartenance et projection}

Si les relations constituent le capital social mobilisable, elles n'existeraient pas sans les expériences, les lieux, les supports auxquels elles sont associées.

Temps court objectivement, temps long dans la mémoire, les « vacances » ou « activités extraordinaires » occupent dans le souvenir des anciens placés enquêtés un espace démesuré. Si les souvenirs de vacances (colonies de vacances, transferts) sont unanimement racontés par les personnes interviewées, c'est aussi parce qu'ils ont davantage l'occasion d'être remémorés, par le biais de photos ou du fait qu'ils fassent événement pour un ensemble de personnes - jeunes, mais également professionnels.

«On a eu une enfance très heureuse. En dehors de cela, le mercredi, le week-end, les vacances, notre vie c'était le village entier. On partait en colo, on a visité énormément de choses, on a fait des trucs, que je ne sais pas si une famille ordinaire, ou même moi j'offrirais à mes enfants. On partait en vacances trois fois par an, sans compter les classes vertes de l'école, on faisait des activités encadrées par une équipe éducative, c'était vraiment chouette » (Jessica, 34 ans, ancienne placée en village d'enfants).

Ces expériences, si elles sont créatrices de liens, offrent par ailleurs la possibilité d'inscription dans des groupes d'appartenance parfois plus valorisants que l'école ${ }^{38}$. Ainsi une passion pour les animaux qui s'est développée pendant la période de placement peut perdurer et permettre de rencontrer de nouvelles personnes. Voire de faire de sa passion une activité professionnelle :

« J'ai été éleveur canin. En France, je suis à l'origine d'une nouvelle race que j'ai développée, enfin j'ai promu la race en France, et aujourd'hui, je fais plus d'élevage, mais je suis connu dans ce monde-là en fait, j'ai encore des coups de fil, des mails, je suis vraiment à la base, et aujourd'hui c'est une grosse mode : American Bully ça s'appelle... » (Damien, 34 ans, ancien placé en village d'enfants).

38. Le rapport d'Isabelle Frechon et Annick-Camille Dumaret, "Bilan critique de cinquante ans d'études sur le devenir adulte des enfants placés » (ONED, 2008), fait état des difficultés rencontrées à l'école par les enfants placés, démontrées dans la grande majorité des recherches effectuées. 
Le lien est ici associé au support de lien, soit une identité valorisante et valorisée et un « groupe d'appartenance ${ }^{39}$ » qui a la possibilité d'exister parfois avant le placement, mais surtout pendant et après celui-ci.

Les espaces géographiques - lieux de placement, lieux de formation ou même lieu où les jeunes ont eu l'occasion de partir en transfert constituent également des espaces d'identification et de projection. Parfois, ce sont les lieux que certains jeunes choisissent pour chercher un premier emploi, ou encore le lieu où ils reviennent après un passage éclair chez leurs parents au moment de leur fin de prise en charge.

Ainsi le « capital social ou réseau des liaisons ${ }^{40}$ " se bâtit à partir de loisirs, de passions communes, mais aussi de lieux d'habitation. Autant de supports qui permettent de construire puis de faire fructifier le réseau pendant et après le placement, en attendant que vienne s'ajouter la construction d'un « réseau de liaison ${ }^{41}$ » pour ces jeunes qui, bien souvent, arrêtent les études à la fin de leur prise en charge ou peu après.

\section{POUR CONCLURE}

Dans une société de valorisation d'une rhétorique individualiste de la réussite personnelle, il est difficile de mettre en lumière l'impact du capital social. Pourtant, il est bien au cœur des trajectoires. Les institutions de l'Aide sociale à l'enfance, en se centrant sur l'insertion professionnelle et l'accompagnement individualisé, négligent la dimension du capital social et des collectifs. Les pratiques sportives, culturelles, de loisirs ne sont pas secondaires dès lors qu'elles permettent de faire l'expérience de la rencontre avec l'autre, parfois différent (ne faisant pas partie du groupe d'enfants placés). Ainsi la multiplicité des groupes d'appartenance constitue des occasions de valorisation (pour des jeunes qui ne sont pas nécessairement valorisés à l'école par exemple), d'expérience de pluralité identitaire, évitant l'enfermement dans l'identité d'enfant placé, dans une identité déviante ou extrémiste entre autres.

39. « On appelle groupe d'appartenance le groupe dans lequel l'individu a des relations directes, de face à face avec les autres membres. Le groupe dont il fait partie effectivement et physiquement à tel moment de sa vie » (Alex Mucchielli, L'identité, Paris, Puf, « Que sais-je ?», 1980, p. 99).

40. Pierre Bourdieu, op. cit., p. 2.

41. "L'existence d'un réseau de liaison n'est pas un donné naturel, ni même un "donné social", constitué une fois pour toutes et pour toujours par un acte social d'institution (représenté, dans le cas du groupe familial, par la définition généalogique des relations de parenté qui est caractéristique d'une formation sociale), mais par le produit du travail d'instauration et d'entretien qui est nécessaire pour produire et reproduire des liaisons durables et utiles, propres à procurer des profits matériels ou symboliques » (Bourdieu, op. cit., p. 2). 
Les professionnels ne mesurent pas toujours l'impact de leur travail lorsqu'ils accompagnent un collectif, proposent des activités ou favorisent le maintien d'activités de loisirs pour les jeunes. Nous finirons par un exemple de l'expérimentation de colocation de jeunes sortant de l'Aide sociale à l'enfance. Pour les professionnels, l'expérimentation est un échec car les logements ont été dégradés, les voisins se sont plaints du bruit et il y a eu des conflits entre les jeunes. Du point de vue des jeunes, si la gestion des tâches domestiques a engendré des conflits, ils ont appris les uns des autres, rencontré le réseau de chacun et accédé par cette expérience à une connaissance d'eux-mêmes et des autres. Parce que «l'expérience de la conscience de soi » ne résulte pas « de l'autoréflexion du sujet connaissant sur lui-même, mais de l'expérience de l'interaction ${ }^{42}{ }^{\prime}$, l'autonomie ne peut s'acquérir que par la constitution, le maintien et l'élargissement d'un capital social.

42. Axel Honneth, «La théorie de la reconnaissance: une esquisse», De la reconnaissance. Don, identité et estime de soi, Revue du Mauss, $n^{\circ}$ 23, 2004. 